\title{
Microscopic Black Holes as a Source of Ultrahigh Energy $\gamma$-rays
}

\author{
Roberto Casadio*, Benjamin Harms ${ }^{\dagger}$ and Octavian Micu ${ }^{\dagger}$ \\ ${ }^{*}$ Dipartimento di Fisica, Università di Bologna and I.N.F.N., Sezione di Bologna, via Irnerio 46, \\ 40126 Bologna, Italy \\ ${ }^{\dagger}$ Department of Physics and Astronomy, The University of Alabama, Box 870324, Tuscaloosa, AL \\ 35487-0324, USA
}

\begin{abstract}
We investigate the idea that ultrahigh energy $\gamma$-rays $(E>10 \mathrm{TeV})$ can be produced when charged particles are accelerated by microscopic black holes. We begin by showing that microscopic black holes may exist as remnants of primordial black holes or as products of the collisions in the large extra dimensions scenario of high energy cosmic rays with atmospheric particles. We then solve Maxwell's equations on curved spacetime backgrounds in 4, 5 and 6 dimensions and use the solutions to calculate the energy distributions. From the latter we obtain the black hole parameters needed to produce the energies of the observed $\gamma$-rays.
\end{abstract}

\section{INTRODUCTION}

The existence of cosmological black holes with masses of $10-10^{8}$ solar masses is now an accepted fact. The existence of microscopic black holes with masses of $1-10$ Planck masses has not been established, but their existence is of great theoretical interest. If they do exist, there is the possibility that they could be probed experimentally to obtain information about quantum gravity. One possible source of such microscopic black holes is primordial black holes, which have reached Planck-size masses during the present epoch through emission of Hawking radiation. Another possible source is microscopic black hole production. The recent proposal of the existence of large extra dimensions [1] and the consequent lowering of the fundamental energy scale to $1 \mathrm{TeV}$ implies that microscopic black holes can be created in accelerators [2] whose center of mass energies are above the fundamental energy scale. Microscopic black holes would also be produced in the collisions of ultrahigh energy cosmic rays with the Earth's atmosphere [3]. In this talk we describe one of the signatures of black holes impinging upon the Earth's atmosphere: ultrahigh energy $\gamma$-rays. As of yet there is no firm evidence that such emissions are occurring in our atmosphere, but there is some evidence for $\gamma$-rays with energies greater than $100 \mathrm{TeV}$ at the $1.6 \sigma$-level from unknown sources within the galatic plane[ 4$]$.

A charged particle being accelerated by a black hole can produce $\gamma$-rays with energies in the multi-TeV range before the particle passes beyond the horizon radius provided that the curvature gradient of the space around the black hole is large enough. Such curvature gradients occur in quantum black holes, black holes whose masses are of the order the Planck mass. A calculation taking into account special relativity (but not 
general relativity) shows us that to produce $\gamma$-ray energies in the $10 \mathrm{TeV}$ range a single electronic charge would have to be accelerated by a black hole with a mass equal to five times that of the Planck mass.

The microscopic black holes needed to produce ultrahigh $\gamma$-rays may be the remnants of primordial black holes. Such black holes can be produced by

- Inflationary horizon-scale fluctuations

- Density fluctuations at phase transitions and bubble formation and collapse

- Baryon isocurvature fluctuations on small scales.

Large-mass primordial black holes $\left(M>10^{15} \mathrm{gm}\right)$ decaying via Hawking radiation [5] as described by the canonical ensemble in 4 space-time dimensions, $(d M / d t) \sim-M^{-2}$, would have decayed to a Planck-size mass in the present epoch. Microscopic black holes produced in this manner could be stable if quantum gravity effects terminate the decay process.

Copious microscopic black hole production can also occur if large extra dimensions exist. In this scenario black hole production can occur as the result of the collision of particles with total center of mass energy above the effective Planck scale, which can be as low as the electroweak scale $m_{e w} \sim 1 \mathrm{TeV}$. Black holes could thus be produced in collisions of high energy cosmic rays with the Earth's atmosphere. As we show in the next Section, such black holes may live long enough to create ultrahigh $\gamma$-rays even without taking quantum gravity effects into account.

\section{BLACK HOLES AND LARGE EXTRA DIMENSIONS}

In a 4-dimensional space-time, a black hole might emerge from the collision of two particles only if its center of mass energy exceeds the Planck mass $m_{p}\left(l_{p}\right.$ will denote the Planck length). In fact, the Compton wavelength $l_{M}=l_{p}\left(m_{p} / M\right)$ of a point-like particle of mass $M<m_{p}$ would be smaller than the gravitational radius $R_{H}=2 G_{N} M=$ $2\left(l_{p} / m_{p}\right) M$ and the very (classical) concept of a black hole would lose its meaning. However, since the fundamental mass scale is shifted down to $m_{e w}$ in the models under consideration, black holes with $M \ll m_{p}$ can now exist as classical objects provided

$$
l_{p}\left(m_{p} / M\right) \ll R_{H} \ll L,
$$

where $L$ is the scale at which corrections to the Newtonian potential become effective. The left hand inequality ensures that the black hole behaves semiclassically, and one does not need a full-fledged theory of quantum gravity, while the right hand inequality guarantees that the black hole is small enough that its gravitational field can depart from the Newtonian behavior without contradicting present experiments.

The luminosity of a black hole in $D$ space-time dimensions is given by

$$
\mathscr{L}_{(D)}(M)=\mathscr{A}_{(D)} \int_{0}^{\infty} \sum_{s=1}^{S} n_{(D)}(\omega) \Gamma_{(D)}^{(s)}(\omega) \omega^{D-1} d \omega
$$


where $\mathscr{A}_{(D)}$ is the horizon area in $D$ space-time dimensions, $\Gamma_{(D)}^{(s)}$ the corresponding grey-body factor and $S$ the number of species of particles that can be emitted. For the sake of simplicity, we shall approximate $\sum_{s} \Gamma_{(D)}^{(s)}$ as a constant. The distribution $n_{(D)}$ is the microcanonical number density [6, 7, 8]

$$
n_{(D)}(\omega)=C \sum_{l=1}^{[[M / \omega]]} \exp \left[S_{(D)}^{E}(M-l \omega)-S_{(D)}^{E}(M)\right]
$$

where $[[X]]$ denotes the integer part of $X$ and $C=C(\omega)$ encodes deviations from the area law [6] (in the following we shall also assume $C$ is a constant in the range of interesting values of $M$ ).

\section{ADD scenario}

If the space-time is higher dimensional and the extra dimensions are compact and of size $L$, the relation between the mass of a spherically symmetric black hole and its horizon radius is changed to [9]

$$
R_{H} \simeq l_{(4+d)}\left(\frac{2 M}{m_{(4+d)}}\right)^{\frac{1}{1+d}}
$$

where $G_{(4+d)} \simeq L^{d} G_{N}$ is the fundamental gravitational constant in $4+d$ dimensions. Eq. (4) holds true for black holes of size $R_{H} \ll L$, or, equivalently, of mass $M \ll M_{c} \equiv$ $m_{p}\left(L / l_{p}\right)$. Since $L$ is related to $d$ and the fundamental mass scale $m_{(4+d)}$ by [1]

$$
L \sim \gamma^{1+\frac{2}{d}} 10^{\frac{31}{d}+16} l_{p}
$$

where $\gamma \equiv m_{e w} / m_{(4+d)}$, Eq. (11) translates into

$$
10^{-\frac{31+16 d}{2+d}} \gamma m_{p} \sim 10^{-16} \gamma m_{p} \ll M \ll M_{c},
$$

where we also used the fact that $d=1$ is ruled out by present measurement of $G_{N}$ [1]] and relatively high values of $d$ (i.e., $d \sim 6$ ) seem to be favored (see, e.g., Refs. [10]). For $\gamma \sim 1$ (i.e., $m_{(4+d)} \sim m_{e w} \sim 1 \mathrm{TeV}$ ), the left hand side above is of order $m_{e w}$ as well.

For $R_{H}<L$ the Euclidean action $S_{E}^{<} \sim\left(M / m_{e w}\right)^{(d+2) /(d+1)}$ and the occupation number density for the Hawking particles in the microcanonical ensemble is given by

$$
n_{(4+d)}(\omega) \sim \sum_{l=1}^{[[M / \omega]]} e^{\left(\frac{M-l \omega}{m_{e w}}\right)^{\frac{d+2}{d+1}}-\left(\frac{M}{m_{e w}}\right)^{\frac{d+2}{d+1}}}
$$

In 4 dimensions one knows that microcanonical corrections to the luminosity become effective only for $M \sim m_{p}$, therefore, for black holes with $M \gg m_{e w}$ the luminosity (2) 


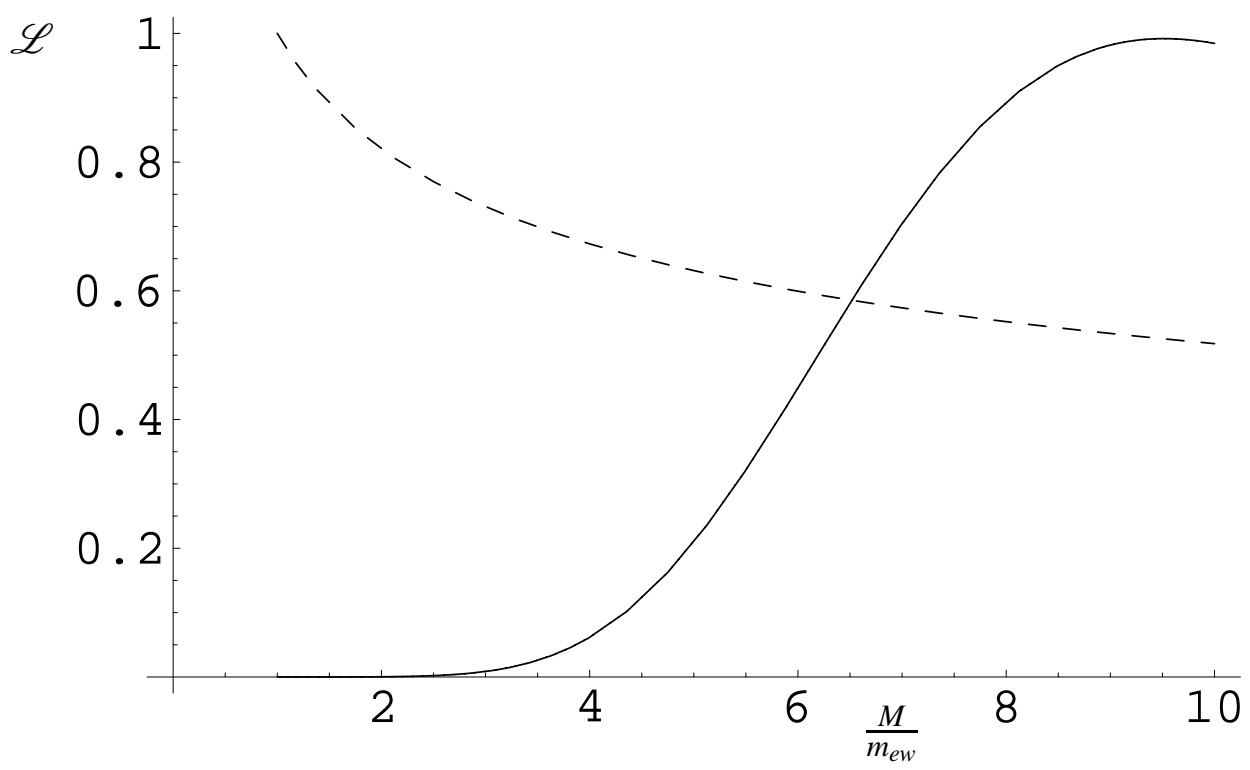

FIGURE 1. Microcanonical luminosity (solid line) for a small black hole with $d=6$ extra dimensions compared to the corresponding canonical luminosity (dashed line). Vertical units are chosen such that the canonical luminosity $\mathscr{L}\left(m_{e w}\right)=1$.

should reduce to the canonical result [11], 12, 13]. However, in $4+d$ dimensions, such corrections are not entirely negligible for $M \sim m_{e w}$. The decay rate corresponding to the number density (7) as calculated from Eq. (2) is exhibited for $d=6$ in Fig. 11. For lower values of $d$ the peak in the microcanonical luminosity shifts to smaller values of $M$, thus approaching the 4-dimensional canonical decay rate (also shown for comparison in Fig. 11). In all cases, the microcanonical luminosity becomes smaller for $M \sim m_{e w}$ than it would be according to the canonical luminosity, which makes the life-time of the black hole somewhat longer than in the canonical picture. In particular, for $d=6$ one finds

$$
\left.\frac{d M}{d t}\right|_{M \sim m_{e w}} \simeq-10^{-10} \mathscr{L}_{(10)}^{H} \sim-10^{17} \frac{\mathrm{TeV}}{\mathrm{s}} .
$$

A black hole would therefore evaporate very quickly [2] down to $\sim 6 m_{e w}$. Then, its lifetime is dominated by the time it would take to emit the remaining $\Delta M \sim 5 \mathrm{TeV}$, before it reaches $1 m_{e w}$, which is approximately

$$
T \sim\left(\frac{d M}{d t}\right)^{-1} \Delta M \sim 10^{-17} \mathrm{~s} .
$$

The above relatively long time also takes into account the dependence of the greybody factor $\Gamma_{(4+d)}^{(s)}$ on $d$ (for the details see Ref. [14]). Without a full-fledged theory of quantum gravity, nothing can be safely stated as $M$ becomes less than $m_{e w}$. However, by extrapolating the microcanonical behavior, one would conclude that the evolution then proceeds roughly according to the usual exponential law of radiative decay [7]. 


\section{RS scenario}

In order to study this case, we shall make use of the solution given in Ref. [15]. This is one of the few known metrics on the brane which might represent such a case in the context of the RS scenario (for more candidates see Ref. [16]). Such a solution has the Reissner-Nordström form

$$
-g_{t t}=\frac{1}{g_{r r}}=1-2 \frac{M l_{p}}{m_{p} r}+Q^{2} \frac{l_{p}^{2}}{r^{2}}-q \frac{m_{p}^{2} l_{p}^{2}}{m_{(5)}^{2} r^{2}},
$$

and the (outer) horizon radius is given by

$$
R_{H}=l_{p} \frac{M}{m_{p}}\left[1+\sqrt{1-Q^{2} \frac{m_{p}^{2}}{M^{2}}+\frac{q m_{p}^{4}}{M^{2} m_{(5)}^{2}}}\right]
$$

where $m_{(5)} \sim m_{e w}$ is the fundamental mass scale and $q$ represents a (dimensionless) tidal

charge. The latter can be estimated on dimensional grounds as [12, 15] $q \sim\left(\frac{m_{p}}{m_{e w}}\right)^{\alpha} \frac{M}{m_{e w}}$ and for $\alpha>-4$ the tidal term $\sim 1 / r^{2}$ dominates over the 4-dimensional potential $\sim 1 / r$ (as one would expect for tiny black holes). From Eq. (11) with $Q=0$ and $\alpha>-4$ one obtains

$$
R_{H} \simeq l_{p}\left(\frac{m_{p}}{m_{(5)}}\right)^{1+\frac{\alpha}{2}} \sqrt{\frac{M}{m_{(5)}}},
$$

since the tidal term $q$ dominates for both $M$ and $m_{(5)} \ll m_{p}$, and one must still have Eq. (II). With one warped extra dimension [[17], the length $L$ is just bounded by requiring that Newton's law not be violated in the tested regions, since corrections to the $1 / r$ behavior are of order $(L / r)^{2}$. This roughly constrains $l_{p}<L<10^{-3} \mathrm{~cm}$. Hence the allowed masses are, according to Eq. (11),

$$
\left(\frac{m_{(5)}}{m_{p}}\right)^{\frac{\alpha}{3}} \ll \frac{M}{m_{(5)}} \ll\left(\frac{L}{l_{p}}\right)^{2}\left(\frac{m_{(5)}}{m_{p}}\right)^{2+\alpha} .
$$

In particular one notices that black holes with $M \sim m_{(5)} \sim m_{e w}$ could exist only if the following two conditions are simultaneously satisfied

$$
\alpha \geq 0 \text { and } \frac{L}{l_{p}} \gg\left(\frac{m_{p}}{m_{e w}}\right)^{\frac{3+\alpha}{3}} .
$$

The luminosity is now given by, for the limiting case $\alpha=0$ and taking into account the second condition in Eq. (14),

$$
\mathscr{L}_{(4)}<10^{-9} \frac{M}{m_{e w}} \frac{\mathrm{TeV}}{\mathrm{s}}
$$

which yields an exponential decay with typical life-time $T>10^{9} \mathrm{~s}$. 


\section{Production of Black Holes by Cosmic Rays}

Since the Planck mass in $4+d$ dimensions can be as small as $1 \mathrm{TeV}$, black holes can be created in cosmic ray interactions with particles in the Earth's atmosphere (or any other source of matter within the galaxy) in the processes

$$
\begin{aligned}
& p+p \rightarrow \mathrm{BH}+X^{++} \\
& v+N \rightarrow \mathrm{BH}+X .
\end{aligned}
$$

The cross section for the production of a black hole with mass $M$ in such processes is given to a good approximation by $\sigma \simeq \pi R_{H}^{2}$, where

$$
R_{H}=\frac{1}{\sqrt{\pi} m_{(4+d)}}\left[\frac{M}{m_{(4+d)}}\left(\frac{8 \Gamma\left(\frac{d+3}{2}\right)}{d+2}\right)\right]^{\frac{1}{d+1}}
$$

and we recall that $m_{(4+d)} \sim m_{e w}$. For proton and neutrino energies above $\sim 10^{8} \mathrm{TeV}$ black hole production will dominate over the production of standard model particles [18]. About 100 black holes per year are created for the whole surface of the Earth by the $p+p$ process [3], while the $v+N$ process creates one black hole per year [[18].

\section{ACCELERATION OF A CHARGED PARTICLE BY A NEUTRAL BLACK HOLE}

\section{4-Dimensional Case}

To describe the radiation a charged particle being accelerating by a neutral microscopic black hole will produce, Maxwell's equations must be solved on a curved spacetime background. The perturbed electromagnetic tensor elements $f_{\mu \nu}$ are determined from the relation

$$
\left(\sqrt{-g} f^{\mu v}\right)_{, v}=4 \pi \sqrt{-g} j^{\mu}
$$

where $j^{\mu}$ is the current associated with the falling charge. In 4 dimensions the Schwarzschild metric is spherically symmetric (in this Section the masses are in units of length, i.e. $G_{N}=1$ ),

$$
d s^{2}=-\left(1+\frac{2 M}{r}\right) d t^{2}+\left(1+\frac{2 M}{r}\right)^{-1} d r^{2}+r^{2}\left(d \theta^{2}+\sin ^{2} \theta d \phi^{2}\right)
$$

and, in order to solve the set of equations in Eq. (17) the perturbations $f_{\mu \nu}$ are conveniently expanded in tensor harmonics [19]. Using the field equations all of the tensor elements $f_{\mu \nu}$ can be put in terms of a single element, say $f_{12}$. After taking the Fourier 
transform $\left(\frac{\partial}{\partial t} \rightarrow i \omega\right)$ the remaining element satisfies the equation

$$
\frac{d^{2} f_{l m}}{d r_{*}^{2}}+\left\{\omega^{2}-\mathrm{e}^{v}\left[\frac{l(l+1)}{r^{2}}\right]\right\} f_{l m}=2 \sqrt{l+1 / 2} \mathrm{e}^{i \omega T} \mathrm{e}^{v}\left[\mathrm{e}^{v} u-\frac{d}{d r}\left(\mathrm{e}^{v} w\right)\right]
$$

where $r_{*}$ is the standard "turtle" coordinate,

$$
\begin{gathered}
f_{l m}=\mathrm{e}^{v} f_{12} \\
\mathrm{e}^{v}=1-\frac{2 M}{r}, \quad u=\frac{q \mathrm{e}^{-v}}{r^{2}}, \quad w=\frac{q}{l(l+1)} \frac{d T}{d r} \\
T=-4 M \sqrt{r /(2 M)}-(4 / 3) M[r /(2 M)]^{3 / 2}-2 M \ln (\sqrt{r /(2 M)}-1) \\
+2 M \ln (\sqrt{r /(2 M)}+1) .
\end{gathered}
$$

The charge on the infalling particle is $q$ and $T$ is the time for the particle to fall from $\infty$ to the point $r$. The equation for $f_{l m}$ cannot be solved analytically, but values for the amplitude can be obtained numerically using the Green's function $G\left(r, r^{\prime}\right)$. The solution of Eq. (19) is given by

$$
f_{l m}=\int G\left(r, r^{\prime}\right) S\left(r^{\prime}\right) d r^{\prime}
$$

where $S(r)$ is the source term on the right hand side of Eq. (19). The energy distribution averaged over the complete solid angle is

$$
\left\langle\frac{d E}{d \omega}\right\rangle=\frac{l(l+1)}{2 \pi} f_{l 0} f_{l 0}^{*} .
$$

This distribution is plotted as a solid line in Fig. 2 for unit charge $q$ and black hole mass $M\left(R_{H}=2 M\right)$. The energy of the radiation is

$$
\Delta E \simeq \frac{C_{(4)} q^{2}}{M}
$$

where $C_{(4)}$ is the area under the curve. To produce a $100 \mathrm{TeV}$ photon from a singly charged particle $(q=e)$ the black hole would have to have a mass of approximately $10 m_{p}$.

\section{4+d-Dimensional Case}

For the case of large extra dimensions the invariant line element is taken to be of the form

$$
\begin{aligned}
d s^{2}= & -\left[1-\left(\frac{R_{H}^{2}}{r^{2}+\sum y_{i}^{2}}\right)^{\frac{d+1}{2}}\right] d t^{2}+\left[1-\left(\frac{R_{H}^{2}}{r^{2}+\sum y_{i}^{2}}\right)^{\frac{d+1}{2}}\right]^{-1} d r^{2} \\
& +r^{2}\left(d \theta^{2}+\sin ^{2} \theta d \phi^{2}\right)+\sum d y_{i}^{2}
\end{aligned}
$$




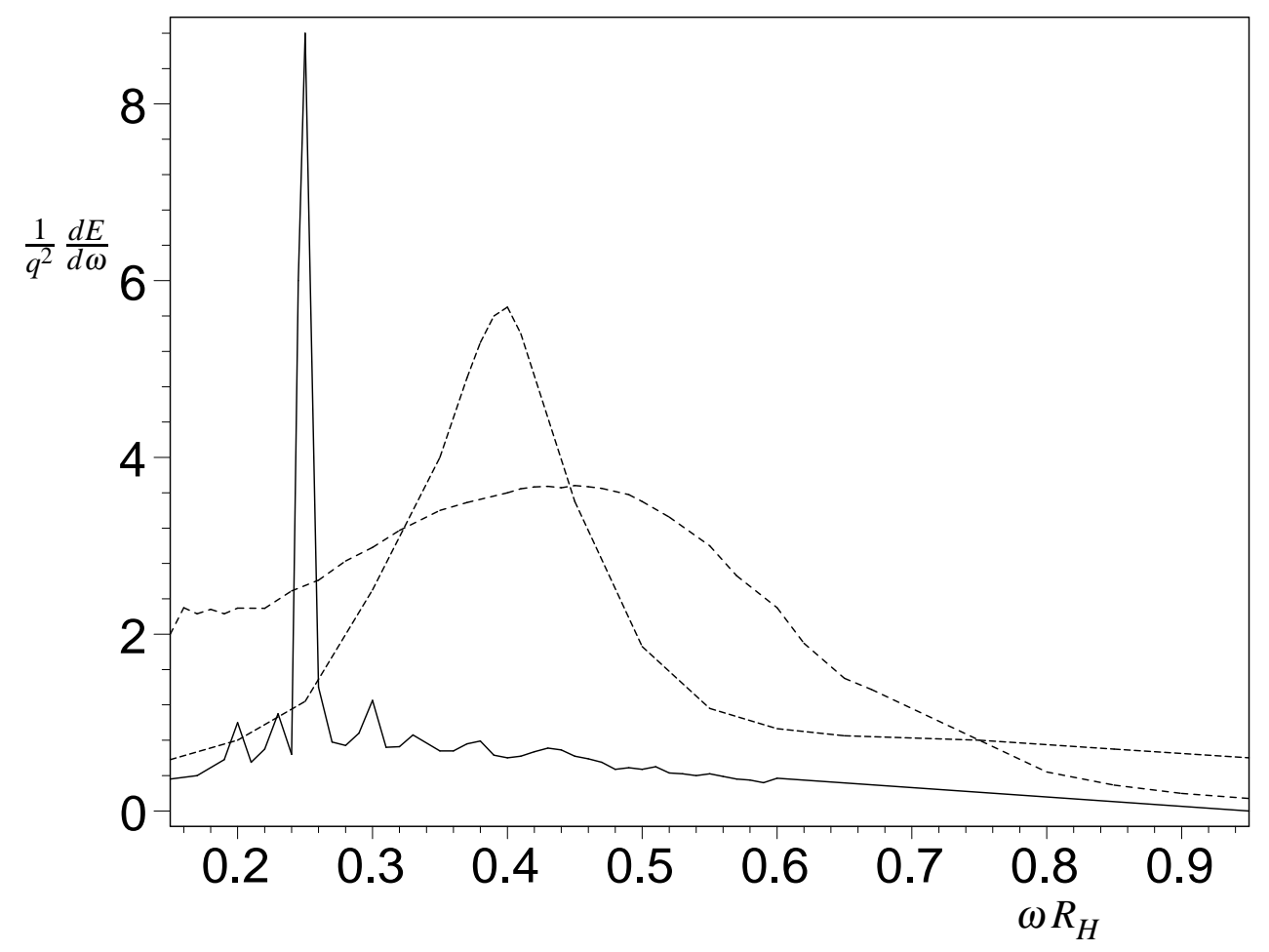

FIGURE 2. Energy distribution for a particle of unit charge falling into a 4-D black hole of mass $M$ (solid line). Same distribution (scaled by 100) for a 5-D black hole (dashed line) and (scaled by 1000) for a 6-D black hole (dotted line). Note that secondary peaks are due to limited numerical precision.

where the $y_{i}$ 's are the coordinates of the $d$ extra dimensions. We assume in this case that the electromagnetic radiation is confined to a 4-dimensional brane embedded in the $4+d$-dimensional space. Thus the $y_{i}$ 's are set to zero in $g_{00}$ and $g_{11}$, and the harmonic tensor has the same form as in Ref. [19], but with extra rows and columns of zeros for the extra dimensions. The energy distributions for the $d=1,2$ cases are given in Fig. 2 together with the 4-dimensional case. The corresponding energy expressions are of the same form as for the 4-dimensional case [Eq. (25)]. It is clear from the graphs that heights of the peaks decrease, that the peaks broaden and that the centers of the peaks shift to larger values of the frequency $\omega$ with increasing number of extra dimensions. The expressions used for the metric tensor elements for the $d>0$ cases are approximations which are valid if $R_{H} \gg L$. The energy radiated in these cases is given by

$$
\Delta E \simeq \frac{C_{(D)} q^{2}}{R_{H}} \quad D=5,6,
$$

where the $C_{(D)}$ 's are twice the areas under the corresponding curves.

The approximation used to obtain the expressions for the 5- and 6-dimensional cases is not satisfactory for $R_{H} \ll L$. The latter is the more realistic case for microscopic black holes, but there is no known metric which suitably describes this case. Therefore the 
problem of obtaining black hole parameters from the observed energies of the $\gamma$-rays remains to be solved.

\section{ACKNOWLEDGMENTS}

This work was supported in part by the U.S. Department of Energy under Grant no. DEFG02-96ER40967.

\section{REFERENCES}

1. N. Arkani-Hamed, S. Dimopoulos and G. Dvali, Phys. Lett. B 429, 263 (1998); Phys. Rev. D 59, 0806004 (1999); I. Antoniadis, N. Arkani-Hamed, S. Dimopoulos and G. Dvali, Phys. Lett. B 436, 257 (1998).

2. S. Dimopoulos and G. Landsberg, Phys. Rev. Lett. 87 (2001) 161602.

3. S. B. Giddings, hep-th/0110127

4. A. Borione, et al, Ap. J. 493, 175 (1998).

5. S.W. Hawking, Nature (London) 248, 30 (1974); Commun. Math. Phys. 43, 199 (1975).

6. B. Harms and Y. Leblanc, Phys. Rev. D 46, 2334 (1992); Phys. Rev. D 47, 2438 (1993); Ann. Phys. 244, 262 (1995); Ann. Phys. 244, 272 (1995); Europhys. Lett. 27, 557 (1994); Ann. Phys. 242, 265 (1995); P.H. Cox, B. Harms and Y. Leblanc, Europhys. Lett. 26, 321 (1994).

7. R. Casadio, B. Harms and Y. Leblanc, Phys. Rev. D 571309 (1998); R. Casadio and B. Harms, Phys. Rev. D 58, 044014 (1998); Mod. Phys. Lett. A17, 1089 (1999).

8. J.M. Bardeen, B. Carter, S.W. Hawking, Commun. Math. Phys. 31, 161 (1973); G.W. Gibbons and S.W. Hawking, Phys. Rev. D 15, 2752 (1977).

9. R.C. Myers and M.J. Perry, Ann. Phys. 172, 304 (1986).

10. S. Cullen, M. Perelstein, Phys. Rev. Lett. 83, 268 (1999); V. Barger, T. Han, C. Kao and R.J. Zhang, Phys. Lett, B 461, 34 (1999); M. Fairbairn, Phys. Lett. B 508 (2001) 335.

11. R. Casadio and B. Harms, Phys. Lett. B 487 (2000) 209.

12. R. Casadio and B. Harms, Phys. Rev. D 64 (2001) 024016.

13. R. Emparan, G.T. Horowitz and R.C. Myers, Phys. Rev. Lett. 85 (2000) 499.

14. R. Casadio and B. Harms, hep-th/0110255.

15. N. Dahdhich, R. Maartens, P. Papadopoulos and V. Rezania, Phys. Lett. B 487, 1 (2000).

16. R. Casadio, A. Fabbri and L. Mazzacurati, gr-qc/0111072; C. Germani and R. Maartens, Phys. Rev. D 64, 124010 (2001).

17. L. Randall and R. Sundrum, Phys. Rev. Lett. 83, 3370 (1999); Phys. Rev. Lett. 83, 4690 (1999).

18. A. Ringwald and H. Tu, Phys. Lett. B525, 135 (2002).

19. F. Zerilli, Phys. Rev. D 9, 860 (1974); M. Johnston, R. Ruffini, F. Zerilli, Phys. Lett. B49, 185 (1974). 